\title{
Immunogold Labeling of Cultured Cells and Virus Particles for Electron Microscopy and Cryo-Electron Microscopy Applications.
}

\author{
Hong $\mathrm{Yi}^{1}$, Joshua D. Strauss ${ }^{2}$, Jason E. Hammonds ${ }^{2}$, Ravi Dyavar Shetty ${ }^{3}$, Rama R. Amara ${ }^{3}$, Paul W. \\ Spearman $^{2}$ and Elizabeth R. Wright ${ }^{1,2}$ \\ 1. Robert P. Apkarian Integrated Electron Microscopy Core, Emory University, Atlanta, Georgia, USA \\ 2. Department of Pediatrics, Emory University School of Medicine, Children's Healthcare of Atlanta, \\ Atlanta, Georgia, USA \\ 3. Department of Microbiology and Immunology, Emory University, Atlanta, Georgia, USA
}

Cryo-electron microscopy (cryo-EM) and cryo-electron tomography (cryo-ET) were first realized in the early 1980s [1] and mid-1990s [2, 3]. Since then both methods have quickly become a powerful tools in structural biology. The major advantage is that they allow for the acquisition of images of biological specimens persevered in their native state after they are plunge-frozen onto carbon-coated grids. When a high voltage transmission electron microscope is used to image the specimen, the resolution reaches the molecular level, which permits the visualization of structures that have not been seen with the conventional electron microscopy. During the past thirty years, the focus has been mainly on ultrastructural analysis, with limited success in the development and application of compatible labeling techniques for assessing the localization of macromolecules. One obstacle for immunogold labeling of frozen-hydrated specimens is the maintenance of specimen viability and structural integrity during the process of immunogold labeling. Most current immunogold procedures require gentle chemical fixation followed by several incubations in buffered solutions. Application of these procedures potentially alters specimen structure.

In this abstract, we introduce a procedure that permits the immunogold labeling of surface antigens of virus particles and of live cells cultured in a monolayer. In this procedure, purified virus particles were immunogold labeled either in suspension or directly on the EM grid using monoclonal primary antibodies and gold particles conjugated to a single Fab fragment just prior to conventional preparation or plunge freezing. For cells cultured on carbon-coated grids, antibodies were added to the culture medium and the incubation was carried out in a $37{ }^{\circ} \mathrm{C}$ incubator. After immunolabeling, the carbon-coated grids with cells will be plunge frozen and then examined with a JEOL JEM-2200FS FEG-TEM operated at $200 \mathrm{kV}$. In our preliminary experiments testing immunogold labeling of live cells, HIV-1 transfected cells were immunogold labeled for tetherin or human CD40 ligand (CD40L). Tetherin is a protein that forms a proteinaceous tether to restrict the release of certain enveloped viruses following viral budding. CD40L is a protein primarily expressed on activated $\mathrm{T}$ cells. After the immunogold labeling was completed, cells were fixed, dehydrated, and embedded. The examination by conventional TEM imaging revealed improved preservation of cellular ultrastructure and abundant immunogold labeling for either tetherin (Figure 1) or CD40L (Figure 2).

We compared the tetherin labeling of live cells with the labeling pattern of chemically fixed cells and did not detect any differences. It should be pointed out that capping and patching has been reported in late 1970s for some membrane proteins when bivalent binding molecules such as immunoglobulin were applied to the live cells $[4,5]$. We concluded that capping and patching did not take place in our labeling strategy. However, additional controls will be included in future labeling experiments. 


\section{References:}

[1] M Adrian, J Dubochet, J Lepault, and AW McCowall, Nature. 308 (1984): 32-36

[2] GJ Jenson and A Briegel, Current Opinion in Structural Biology. 17 (2007): 260-267

[3] K Dierksen, D Typke, R Hegerl, W Baumeister, Ultramicroscopy. 49 (1993): 109-120.

[4] A Ferrante and YH Thong, International Journal for Parasitology. 9 (1979): 599-601

[5] LYW Bourguignon and SJ Singer, Proc. Natl. Acad. Sci. USA. 74 (1977): 5031-5035
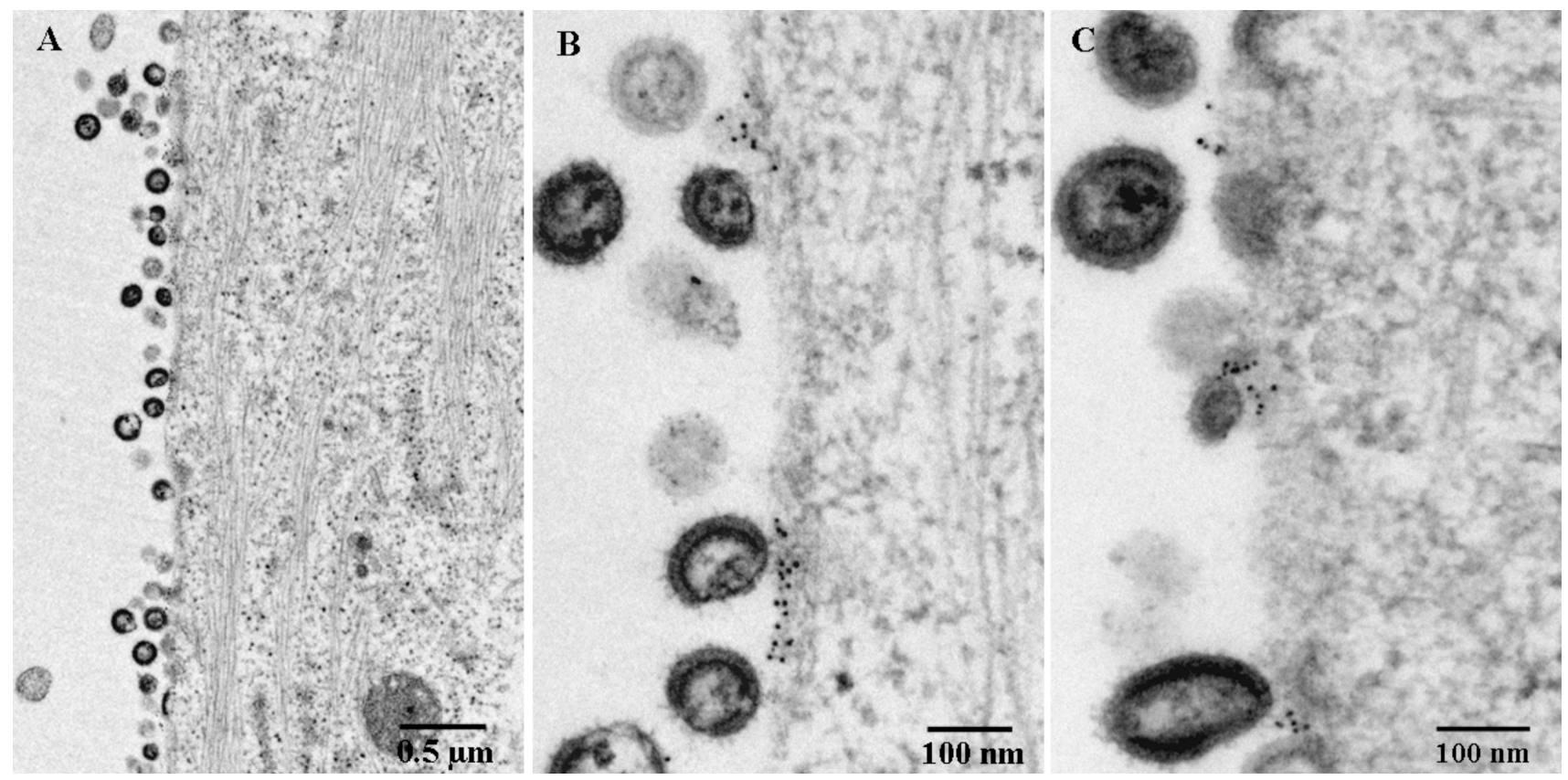

Figure 1. Immunogold labeling of tetherin in HT1080 cells. A: Low magnification image showing HIV-1 VLPs and ultrastructure detail. B and C: Gold particles indicate tetherin localization on filamentous structures between HIV-1 VLPs and cell plasma membrane.
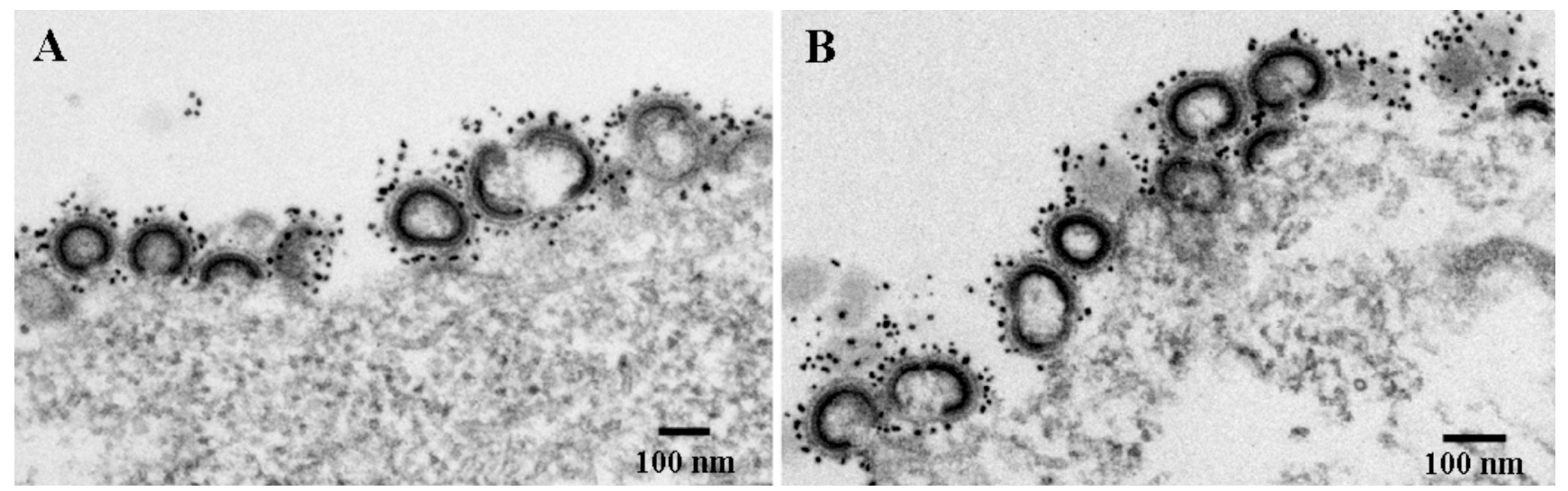

Figure 2. Expression of CD40 ligand (CD154) on HIV-1 VLPs generated from either pIN3-SV40-hCD40L (A) or pIN3-hCD40L (B) HIV/AIDS DNA vaccine transfected 293T cells. 